\title{
Power generation from thermoelectric system-embedded Plexiglas for green building technology
}

\author{
Salman Bin Inayat • Muhammad Mustafa Hussain
}

Received: 31 March 2012/Accepted: 9 June 2012/Published online: 8 July 2012

(C) The Author(s) 2012. This article is published with open access at Springerlink.com

\begin{abstract}
Thermoelectric materials embedded through or inside exterior glass windows can act as a viable source of supplemental power in geographic locations where hot weather dominates. This thermoelectricity is generated because of the thermal difference between the high temperature outside and the relatively cold temperature inside. Using physical vapor deposition process, we experimentally verify this concept by embedding bismuth telluride and antimony telluride through the $5 \mathrm{~mm}$ Plexiglas to demonstrate $10 \mathrm{nW}$ of thermopower generation with a temperature gradient of $21^{\circ} \mathrm{C}$. Albeit tiny at this point with non-optimized design and development, this concept can be extended for relatively large-scale power generation as an additional power supply for green building technology.
\end{abstract}

Keywords Thermoelectricity - Plexiglas ·

Physical vapor deposition - Bismuth telluride .

Antimony telluride

\section{Introduction}

Thermoelectric generation has recently emerged as a significant candidate in the area of renewable and sustainable energy harvesting. With the continuous depletion of fossil fuel reserves, the world has shifted its focus towards renewable energy sources to plan about its future energy requirements. Thermoelectricity like any other renewable energy can come from a natural source capable of

S. B. Inayat · M. M. Hussain ( $\square)$

Integrated Nanotechnology Lab, Electrical Engineering, Physical

Science and Engineering, King Abdullah University of Science

and Technology, Thuwal 2395-6900, Saudi Arabia

e-mail: muhammadmustafa.hussain@kaust.edu.sa replenishing itself over a short span of time. Such a sustainable source being unlimited in reserves, does not have the risk of running out like fossil fuels and have the added advantage of being environment friendly.

Traditionally the efficiency of thermoelectric generation had been as low as $2 \%$, hence restricting its applications to small corners of energy markets. Moreover, with the high cost of thermoelectric materials, commercialization of this technology had been considered financially unfeasible (Song 2008). With the advent of nano-materials and structures synthesis techniques, recent years have seen an increased surge of interest in thermoelectric research and applications.

Systems offering the thermoelectric generation above $10 \%$ are worth pursuing not only because of the amount of fossil fuel saved, but also reducing the hazardous byproducts associated with these conventional sources of energy (Song 2008). Automobiles waste major portion of their energy as exhaust heat, which can be put to advantage by thermoelectric devices placed at suitable spots making maximum use of this wasted heat. Leading car brands like BMW have already launched vehicles employing thermoelectric generators as part of their efficient dynamics technology campaign, claiming a $5 \%$ reduction in fuel consumption (Hall 2009).

Another advantage of thermoelectric devices lies in their long lifetimes and minimum wearing that comes with the moving parts essentially absent in these devices making them highly suitable for deep space applications. NASA has been using radioisotope thermoelectric generators (RTG) in many of their missions (Yang and Caillat 2006).

We have demonstrated, for the first time, a novel design using thermoelectric materials to generate electricity employing thermoelectric conversion using already 
existing differences between outdoor and indoor temperatures. The temperature difference is employed to generate electric power out of thermoelectric generators fabricated through the interface rather than placed on it (Inayat and Hussain 2012). This interface can be a glass window, a brick wall, a wooden door or any number of other materials. These objects would fulfill their blocking purpose with complete integrity, while our power generation model will render them a dual function, making these common objects, harvesters of electric power (Inayat and Hussain 2012). We have already verified and reported (IInayat and Hussain 2012) the viability of a thermoelectric interface on basic metals with minimal TE behavior through a Plexiglas subset of a larger possible interface. Here, we report generation of thermoelectricity due to temperature gradient created across the two faces of a Plexiglas interface slide with specialized TE materials filled through the interface employing a novel deposition technique. The significance of the method lies in the approach adapted to address the challenge of filling up the entire thickness of Plexiglas with physical vapor deposition of thermoelectric material turning it into a power-generating interface. This approach allows thermoelectric materials deposited by sputtering to extend through $5 \mathrm{~mm}$ of Plexiglas panels.

\section{Concept and design}

In 1821, Thomas Seebeck discovered that if different temperature environments $\left(T_{\mathrm{H}}\right.$ and $\left.T_{\mathrm{C}}\right)$ are applied across the two junctions of dissimilar materials coupled together, a potential difference $(\Delta V)$ is created across the junction proportional to the temperature difference $(\Delta T=$ $T_{\mathrm{H}}-T_{\mathrm{C}}$ ). The majority carriers move away from the hot junction towards the cold junction in response to the temperature gradient resulting in a net flow of current through the device on application of an appropriate load. The measure of the generated voltage due to the temperature gradient is termed as thermopower or Seebeck coefficient $S$.

$S=\Delta V / \Delta T$

Performance of thermoelectric materials is evaluated by a unitless figure of merit

$\mathrm{ZT}=\left(\sigma S^{2} / k\right) T$

where, $\sigma$ is the electrical conductivity, $S$ is the Seebeck coefficient and $k$ is the thermal conductivity of the material. In order to increase ZT, engineering the thermal conductivity has been the focus of major thermoelectric research. The thermal conductivity comprises of two parts: the lattice thermal conductivity $k_{\mathrm{L}}$, due to phonon transport; and the electric thermal conductivity, $k_{\mathrm{e}}$, due to the transport of charge carriers. Since electric thermal conductivity is related to electrical conductivity, any attempt to reduce it will also decrease the electrical conductivity of the material, hence compromising the overall ZT of the system.

This leaves us with tampering the lattice thermal conductivity only by scattering the phonons responsible for heat transport through the lattice. Phonon scattering does not alter the electrons transport through the system. Hence research is ongoing to explore various techniques for scattering the phonons as an effective method of reducing the thermal conductivity of thermoelectric materials, and thereby increasing ZT.

Thermoelectric systems have not been employed commonly for mass scale energy generation utilizing the temperature gradients existing between outdoor (environment) and indoor (inside room), due to design limitations (Hudaka and Amatucci 1993). Thermoelectric generators (TEG) can be classified into two broad categories on the basis of their design setup. Conventionally the thermoelectric material or legs are either laid laterally with lateral heat flow through the legs or are placed vertically on a substrate where flow of heat is from top to bottom or vice versa through the device (Fig. 1). Thermoelectric generators manufactured commercially or reported through publications belong to one of these two categories. TEGs reported by Glosch et al. (1999) and Rowe et al. (1989) have their TE legs laid laterally on their respective substrates, whereas those demonstrated by Snyder for JPL (Snyder et al. 2003), Bottner for Micropelt (Bottner et al. 2004), Kishi for Seiko (Kishi et al. 1999) and many others
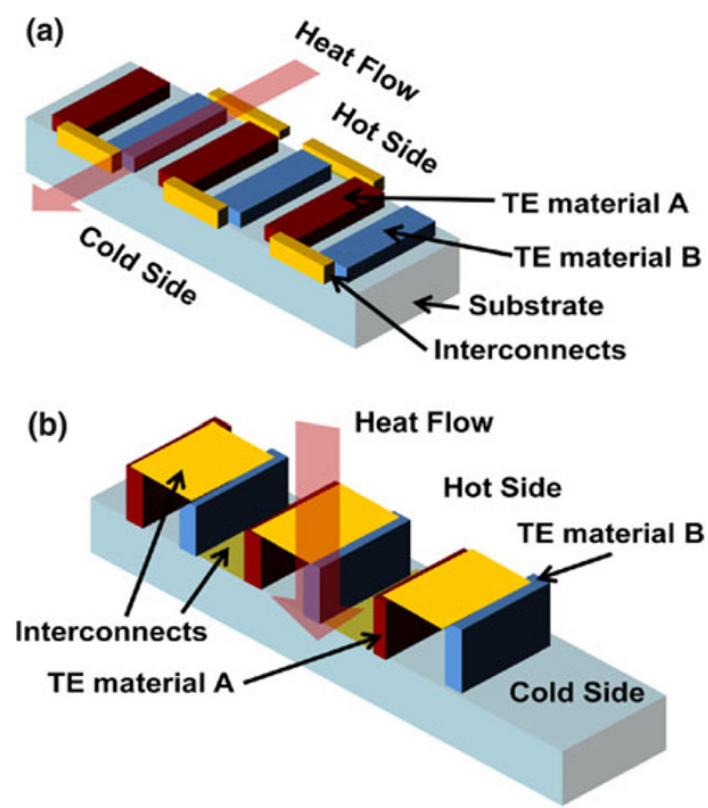

Fig. 1 Conventional thermoelectric generator modules: a lateral b vertical 
have a vertical layout. In both cases the two temperatures are on the same side of the substrate (Fig. 1). The two counter temperature environments we are targeting for our application exist on opposite sides of the blocking interface. By following the conventional design setup and depositing the TE material on either one side of the interface, although allows the temperature environment on that side to access the TE material but prevents the counter temperature of the environment on the opposite side of the interface from influencing the thermoelectric material, hence nullifying the creation of a temperature gradient altogether.

The technique reported in this paper to fill up the entire thickness of an interface with an indirect method is a major step towards transforming full sized domestic windows into thermoelectric interfaces.

\section{Experimental}

In order to realize a power-generating window by depositing thermoelectric materials inside holes drilled in a large glass panel, a novel approach was adapted (Fig. 2). Here, a larger Plexiglas panel was made out of smaller strips of the Plexiglas joined and cured together into a larger panel. Practical demonstration of making a large glass panel with this indirect technique opened up an innovative TE material deposition option. The complimentary TE materials were sputter deposited on the inner sidewalls of the individual strips, such that curing these sidewalls will form the thickness depth of the glass panel with the TE materials effectively embedded through the panel. This technique was successfully implemented for two different types of layouts for TE materials deposition and the approach has
Fig. 2 Process flow for thermoelectric Plexiglas panel cured from individual strips

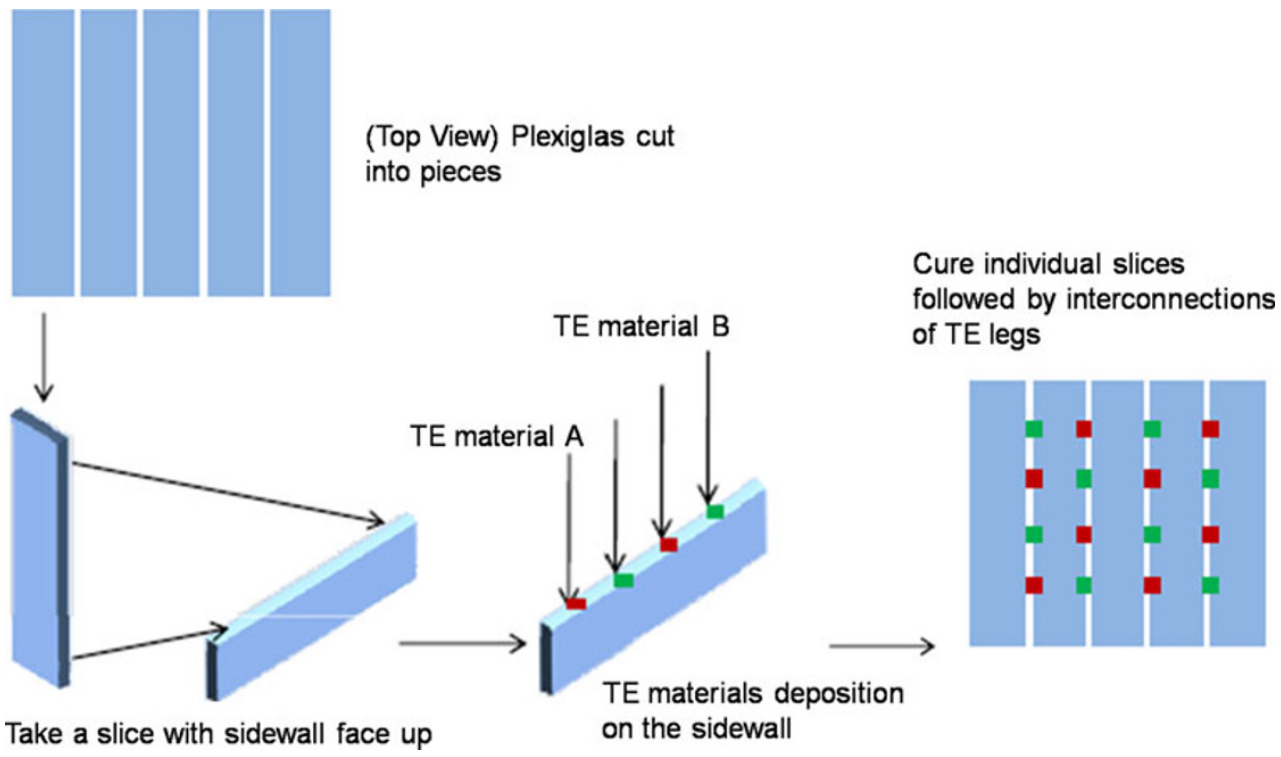

Fig. 3 3-D Schematic of the checkerboard device. Inset shows original checkerboard TE slide

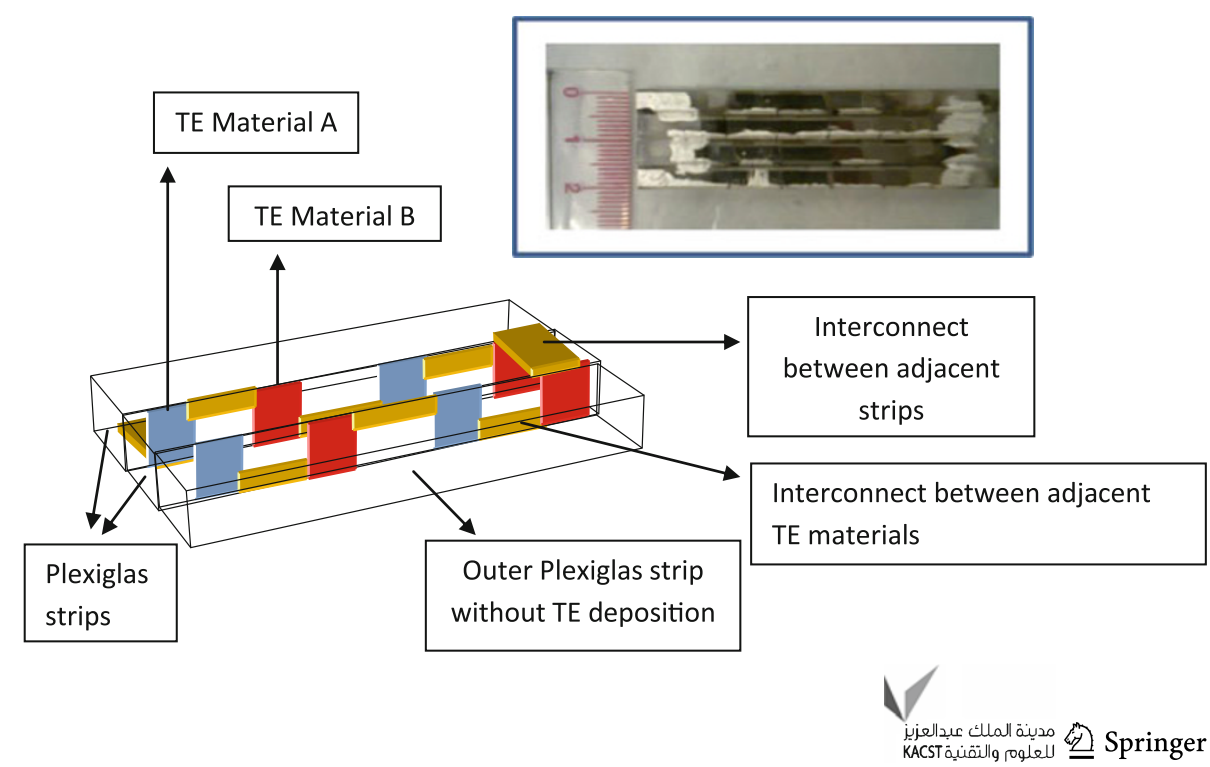


shown promising initial results in terms of output power. We have named the devices as checkerboard and stripe based on the type of layout used for TE material deposition.

A series of 1- $\mu \mathrm{m}$-thick antimony telluride and bismuth telluride blocks $(5 \mathrm{~mm} \times 5 \mathrm{~mm})$ was sputtered using
NEXDEP 120 sputter deposition system in the Integrated Nanotechnology Lab at KAUST on strips of Plexiglas $5 \mathrm{~mm} \times 5 \mathrm{~mm} \times 70 \mathrm{~mm}$. The films were deposited in checkerboard like patterned array of adjacent complimentary TE blocks using a custom-made shadow mask (Fig. 3). The two materials serve as the complimentary thermoelectric
Fig. 4 3-D Schematic of the stripe device. Inset shows original stripe TE slide
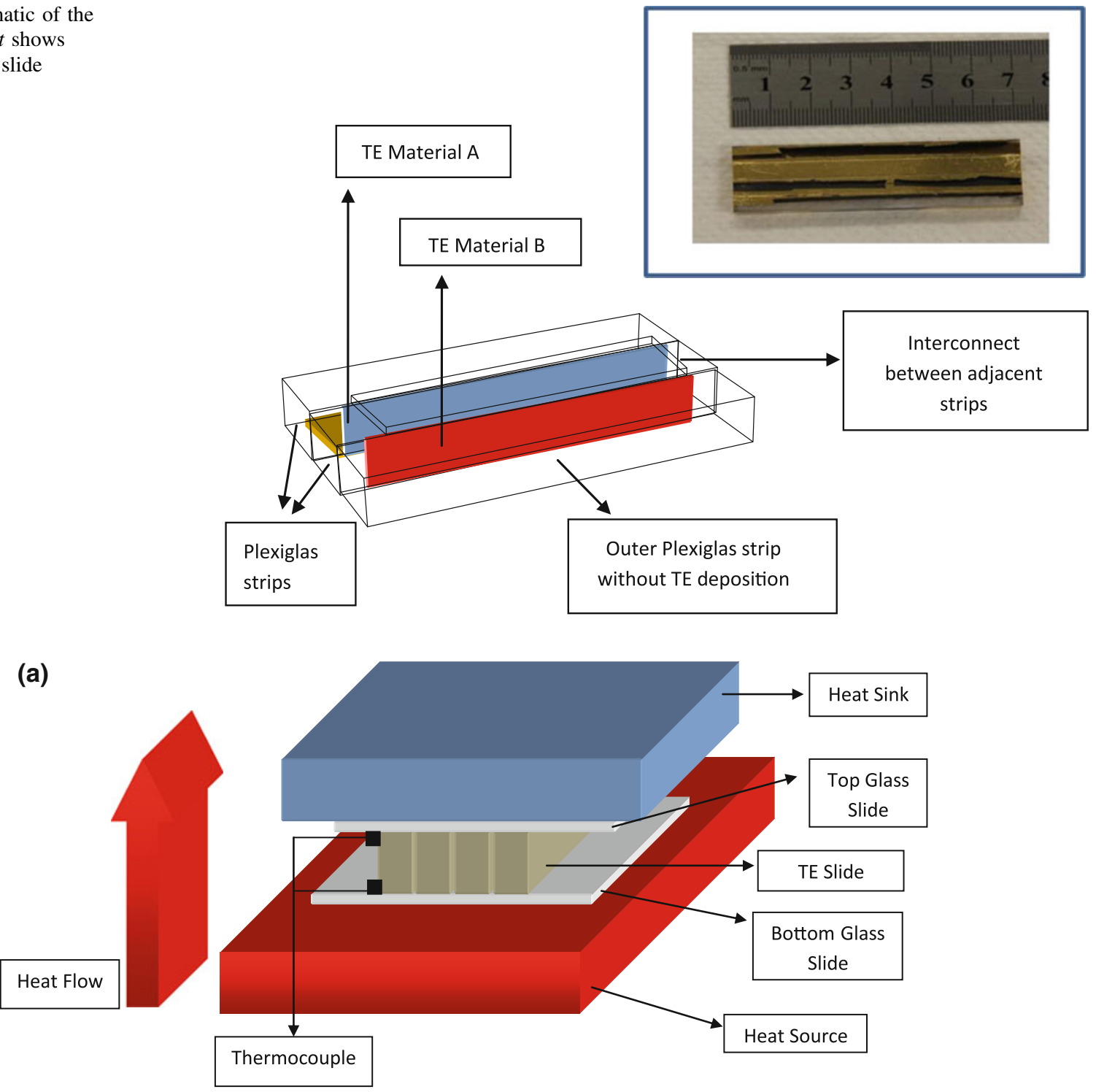

(b)

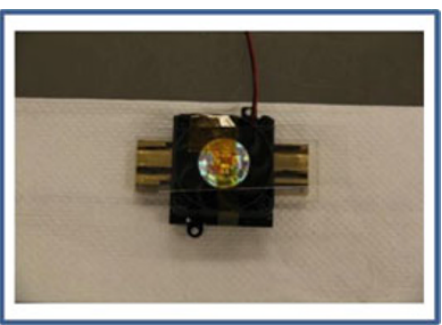

Fig. 5 a 3-D Schematic of the electrical test setup. b TE slide mounted for electrical characterization 
legs, the main functional parts of a thermoelectric generator, embedded through the panel. These individual strips were joined and cured together into a large Plexiglas panel. Interconnections were made between adjacent thermoelectric legs using silver paste. For the second type of device, films of each material were deposited as stripes over the entire length of individual strips, eliminating the need for shadow mask and patterning of the films. On this occasion, $1-\mu \mathrm{m}$-thick antimony telluride and bismuth telluride films were sputtered using NEXDEP 120 sputter deposition system on strips of Plexiglas $5 \mathrm{~mm} \times 5 \mathrm{~mm} \times$ $70 \mathrm{~mm}$. Each strip now serves as a large thermoelectric leg of a particular material type (Fig. 4). These individual strips were joined and cured together into a large Plexiglas panel. Interconnections were made between adjacent strips with sputtered films of $20 \mathrm{~nm}$ of titanium followed by $200 \mathrm{~nm}$ of gold. Titanium (Ti) was sputtered at a DC power of $400 \mathrm{~W}$, pressure of $10 \mathrm{mTorr}$ with substratetarget distance of $180 \mathrm{~mm}$. Gold $(\mathrm{Au})$ sputtering conditions included $300 \mathrm{~W}$ of DC power, a pressure of $10 \mathrm{mTorr}$ and substrate-target separation of $160 \mathrm{~mm}$.

\section{Electrical characterization}

Checkerboard and stripe devices were tested for thermoelectric response by sandwiching the panels between two glass slides in order to facilitate fixing of heat sink and fan on the cold side of the panel (Fig. 5). The entire assembly was mounted on CASCADE Microtech Summit probing station with the hot side glass slide resting on the thermal chuck of the probing station. Temperature of the chuck was ramped up in short intervals with the cooling fan continuously running on the cold side maintaining the cold side temperature close to ambient.

\section{Material calibration}

Seebeck coefficient and electrical conductivity of the deposited films were characterized using measurement setup (Fig. 6). The thermoelectric films of antimony telluride and bismuth telluride with thickness of $1 \mu \mathrm{m}$ were deposited on a glass slide followed by $20 \mathrm{~nm} / 200 \mathrm{~nm}$ sputtered films of $\mathrm{Ti} / \mathrm{Au}$ through a custom-built shadow
Fig. 6 Material calibration test bench: a cross-sectional view, b top view, c wire-bonded calibration device (a)

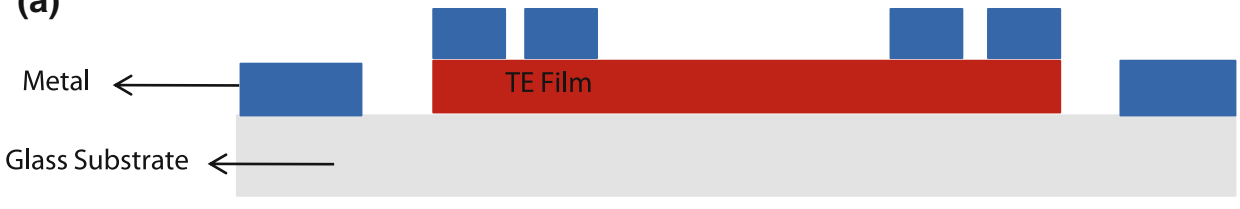

(b)

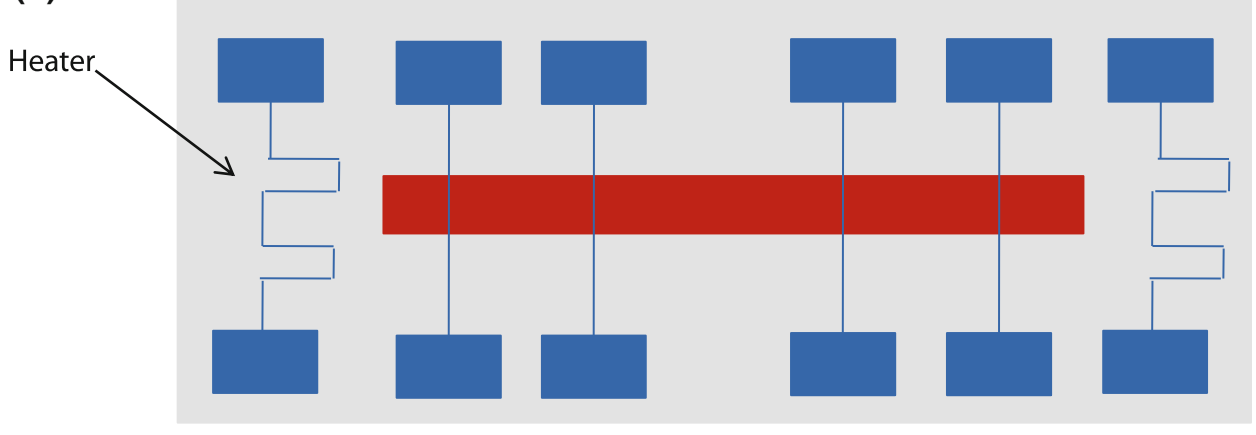

(c)

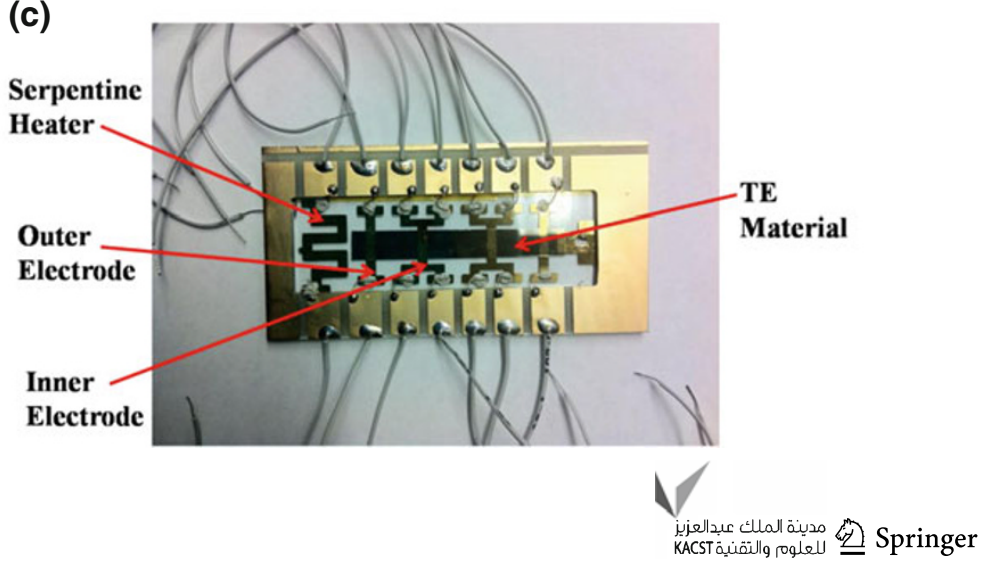


mask to form a set of probing electrodes and a serpentine heater. The slide was wire-bonded for error-free probing. Two copper plates were attached underneath the glass slide. The alignment of the plates was such that one was aligned exactly below the left side inner electrode. This copper plate rested on a hot plate for heating the sample. The second copper plate was aligned exactly below the right side inner electrodes and was placed on a heat sink so that a uniform temperature gradient could be created between the two inner electrodes.

Seebeck voltage was measured across the two inner electrodes for different temperature gradients. Electrical conductivity for the TE thin films was calculated by driving a constant current through the outer electrodes and voltage measured across the two inner electrodes.

\section{Results and discussions}

Generation of thermoelectric voltage in response to applied temperature difference on the two sides of the Plexiglas panel verified the $p$ and $n$ type nature of antimony telluride and bismuth telluride films, respectively. This has also successfully established that reasonable power can be generated by embedding thermoelectric materials through glass panels and subjecting these panels to a temperature difference comparable to solar ambient temperature gradient. Most importantly, our indirect approach of curing a larger Plexiglas panel from individual strips has demonstrated that the entire thickness depth ( $5 \mathrm{~mm}$ in this case) of a domestic window has been filled up with thermoelectric materials using physical vapor deposition (PVD), that could not be achieved otherwise, due to physical limits of vertical deposition by PVD, electrochemical deposition, etc. Neither of these techniques has been known to deposit TE materials as thick as $5 \mathrm{~mm}$, whereas we have used simple PVD to extend the TE material through the entire thickness of the interface.

In case of stripe deposition version of the thermoelectric slide, interconnections were made with sputtered gold films resulting in internal resistance of the generator as low as $6 \Omega$ at room temperature. The output power (Fig. 7) from this panel was less than the checkerboard TE counterpart, due to lower Seebeck voltage generated. With the checkerboard deposition version of the TE slide, for the temperature gradient of $21^{\circ} \mathrm{C}$, the output voltage of the thermoelectric Plexiglas panel was $17.7 \mathrm{mV}$. The interconnections were made with silver paste resulting in a high internal resistance of the generator. Even with the high resistance of $7 \mathrm{k} \Omega$ the output power was greater than the full length counterpart. Promising higher output power levels will be achieved from this Plexiglas panel by improving the internal resistance using gold sputtered interconnections. The inactive to active area ratio of the Plexiglas slides was
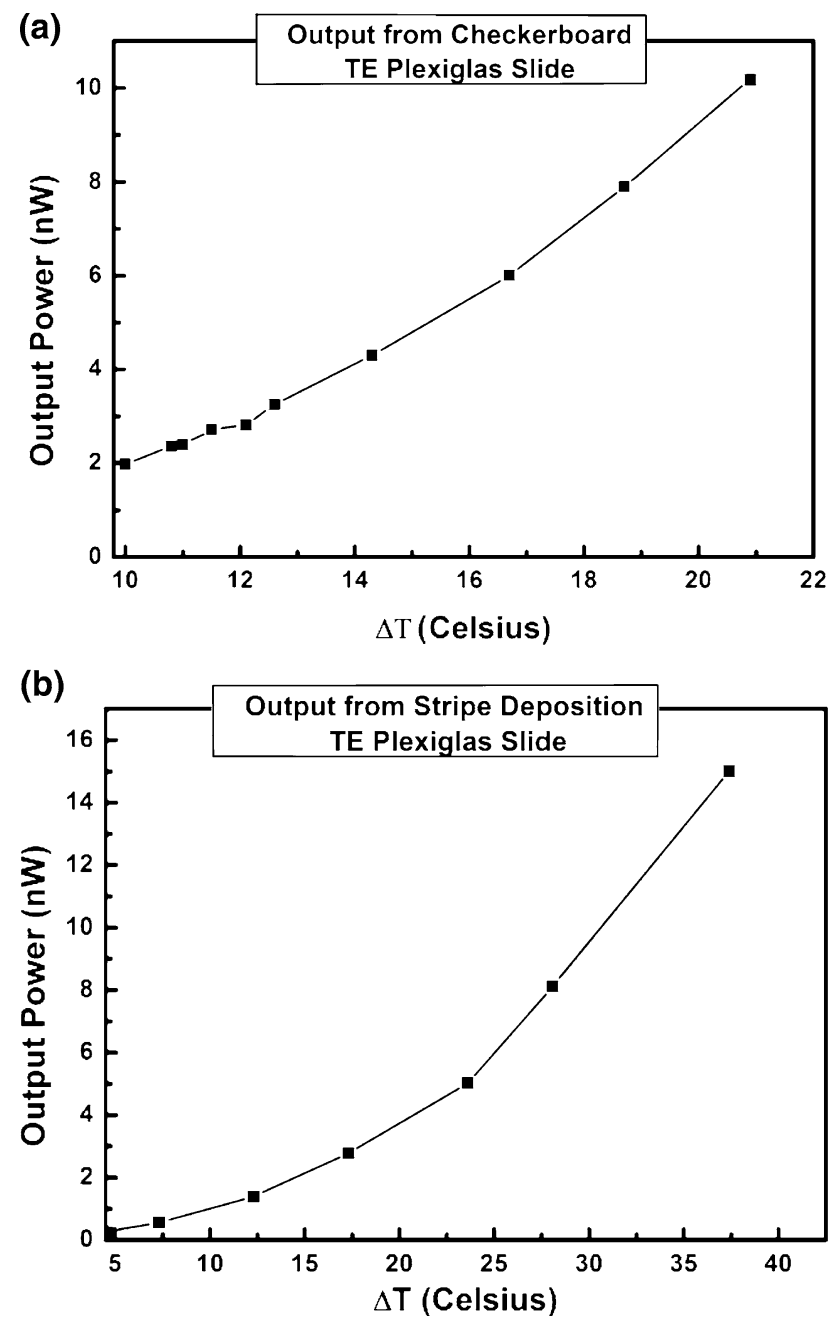

Fig. 7 Output power from: a checkerboard, b stripe TE device

5,000. The ratio was chosen to keep the individual strips wide enough for ease of handling. The power density (power/ $\mathrm{cm}^{2}$ ) of the slide will be considerably increased by reducing the width of individual strips since greater number of strips will be accommodated in the same area.

Thermoelectric characteristics of the deposited films are shown in (Fig. 8). The Seebeck coefficient for antimony telluride and bismuth telluride sputtered thin films was 240 and $-37 \mu \mathrm{V} / \mathrm{K}$, respectively at $314 \mathrm{~K}$. The electrical resistivity for antimony telluride and bismuth telluride thin films was measured to be 638 and $2.22 \mu \Omega \mathrm{m}$, respectively at $304 \mathrm{~K}$. The power factor (PF) being another useful performance barometer was measured to be $9.02 \times 10^{-2}$ and $0.6 \mathrm{~mW} / \mathrm{K}^{2} \mathrm{~m}$ for antimony telluride and bismuth telluride, respectively.

The properties of the films will be further improved by reducing the pressure during sputtering. Under lower pressure the mean free path of the particles is longer and the films landing with higher energies tend to be more dense resulting in relatively lower resistivity values (Bourgault et al. 2008). 

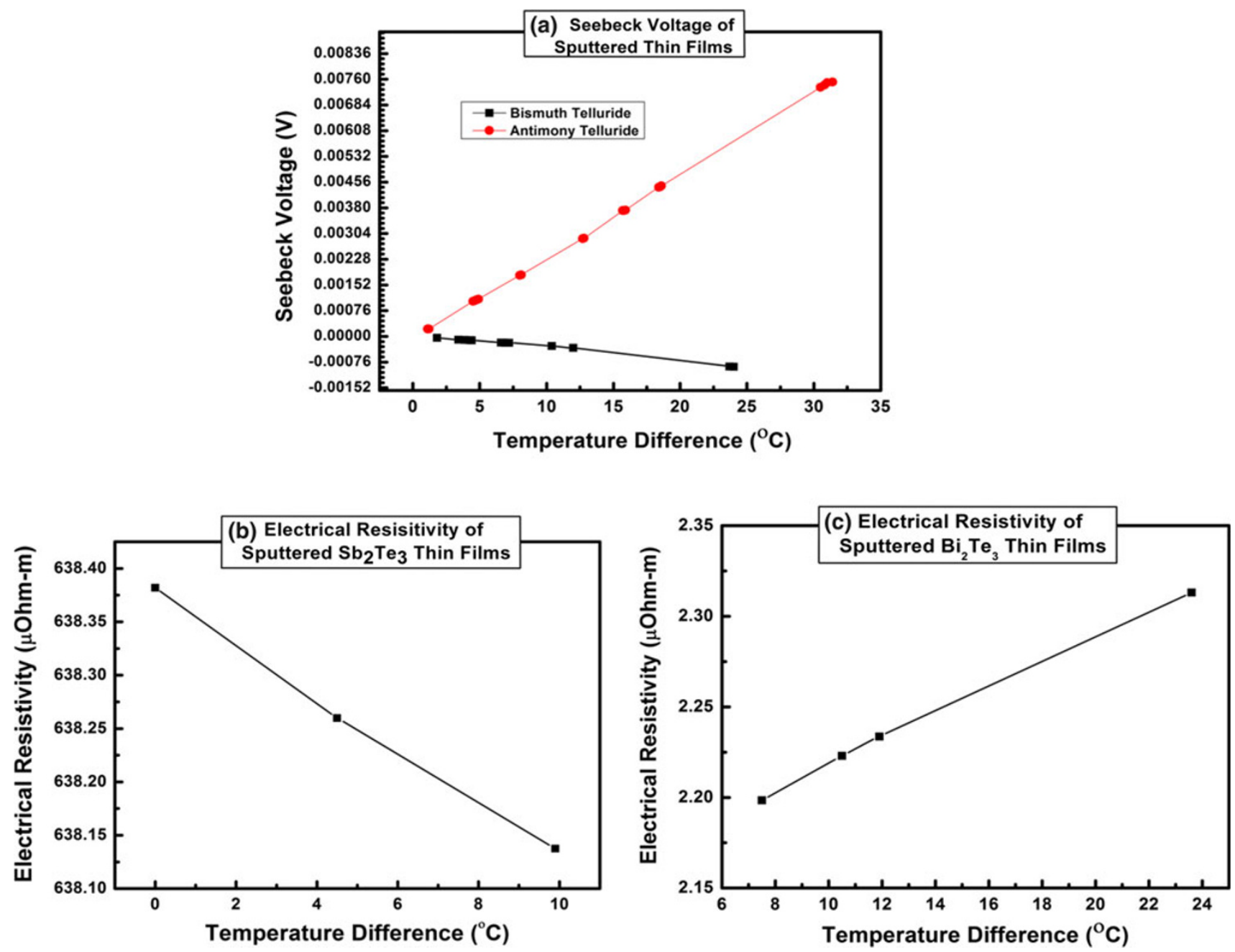

Fig. 8 Thermoelectric properties of sputtered thin films: a Seebeck coefficient of $\mathrm{Sb}_{2} \mathrm{Te}_{3}$ and $\mathrm{Bi}_{2} \mathrm{Te}_{3}, \mathbf{b}$ electrical resistivity of $\mathrm{Sb}_{2} \mathrm{Te}_{3}$, c electrical resistivity of $\mathrm{Bi}_{2} \mathrm{Te}_{3}$

Table 1 Comparison of the thermoelectric properties of $\mathrm{Sb}_{2} \mathrm{Te}_{3}$ thin film with previous reports

\begin{tabular}{llcr}
\hline & $\begin{array}{l}\text { Seebeck } \\
\text { coefficient, } \\
S(\mu \mathrm{V} / \mathrm{K})\end{array}$ & $\begin{array}{l}\text { Electrical } \\
\text { resistivity, } \\
\rho(\mu \Omega \mathrm{m})\end{array}$ & $\begin{array}{l}\text { Power factor, } \\
\mathrm{PF}\left(\mathrm{mW} / \mathrm{K}^{2} \mathrm{~m}\right)\end{array}$ \\
\hline This work & 240 & 638 & $9.02 \times 10^{-2}$ \\
Bourgault et al. (2008) & 304 & 4,500 & $2.6 \times 10^{-2}$ \\
\hline
\end{tabular}

Table 2 Comparison of the thermoelectric properties of $\mathrm{Bi}_{2} \mathrm{Te}_{3}$ thin film with previous reports

\begin{tabular}{llll}
\hline & $\begin{array}{l}\text { Seebeck } \\
\text { coefficient, } \\
S(\mu \mathrm{V} / \mathrm{K})\end{array}$ & $\begin{array}{l}\text { Electrical } \\
\text { resistivity, } \\
\rho(\mu \Omega \mathrm{m})\end{array}$ & $\begin{array}{l}\text { Power } \\
\text { factor, PF } \\
\left(\mathrm{mW} / \mathrm{K}^{2} \mathrm{~m}\right)\end{array}$ \\
\hline This work & -37 & 2.22 & 0.6 \\
Bourgault et al. (2008) & -201 & 340 & 0.12 \\
Huang et al. (2009) & -248 & 7,194 & 0.41 \\
\hline
\end{tabular}

Moreover, at lower pressure there is also a chance of eliminating any presence of oxygen in the films further affecting the electrical resistivity (Khatri and Marsillac 2008).

The thermoelectric output for both types of devices was stable over a long duration of time. Tables 1 and 2 compare the experimental results of deposited $\mathrm{Sb}_{2} \mathrm{Te}_{3}$ and $\mathrm{Bi}_{2} \mathrm{Te}_{3}$ thin films with the thermoelectric characteristics of the previously reported work (Bourgault et al. 2008; Huang et al. 2009). It can be seen from the tables that films deposited in this work are thermoelectrically better (in terms of the PF) than used in the literature (Bourgault et al. 2008; Huang et al. 2009).

\section{Conclusion}

We have demonstrated thermoelectric system-embedded Plexiglas subset for power generation using naturally occurring temperature gradient between outdoor high 
temperature and indoor room ambient environment. Widely used micro-fabrication like PVD has been innovatively employed to extend the thermoelectric material through the entire thickness of Plexiglas successfully dodging/circumventing the physical limits of PVD for vertical deposition. The checkerboard layout version of non-optimized thermoelectric material deposition produced a thermoelectric power in excess of $10 \mathrm{nW}$ on a transparent Plexiglas slide of thickness $5 \mathrm{~mm}$ cured from individual strips of $5 \mathrm{~mm}$ width for an outdoor ambient like temperature gradient. The sidewalls of individual strips were sputtered with complimentary thermoelectric materials to form the thickness depth of the cured panel. The system was tested for artificially created replica of natural temperature gradients between outside (environment) temperature and inside (room) temperature. Thermoelectric properties of the deposited films were measured with an in-house characterization test bench. This power-generating Plexiglas panel serves as a starter for a full-fledged thermoelectric window cured out of individual strips of glass, for energy efficient green buildings. We consider this concept may not be the major power source rather a supplemental source. Thus, we do not need to keep our air conditioning system on to keep it running. It will only be operated when naturally occurring temperature difference exists and can be utilized to power-up or recharge small appliances. Also, this paper reports a proof-of-concept and in future we will chronicle thermoelectric material-based pellet-embedded glass windows as a more practical option.

Acknowledgments We thank Global Research Collaboration Collaborative Fellow Program, King Abdullah University of Science and Technology (KAUST) for the financial grant provided to support this work. We also thank Tayyab Mubeen from KAUST Central Workshop for his logistic support.

Open Access The articles published in this journal are distributed under the terms of the Creative Commons Attribution License which permits any use, distribution, and reproduction in any medium, provided the original author(s) and source are credited.

\section{References}

Bottner H, Nurnus J, Gavrikov A, Kuhner G, Jagle M, Kunzel C, Eberhard D, Plescher G, Schubert A, Schlereth KH (2004) New thermoelectric components using microsystem technologies. J Microelectromech Sys 13:414

Bourgault D, Giroud Garampon C, Caillault N, Carbone L, Aymami JA (2008) Thermoelectric properties of n-type Bi2Te2.7$\mathrm{Se} 0.3$ and p-type Bi0.5Sb1.5Te3 thin films deposited by direct current magnetron sputtering. Thin Solid Films 516:8579

Glosch H, Ashauer M, Pfeiffer U, Lang W (1999) A thermoelectric converter for energy supply. Sens Actuators A 74:246

Huang H, Luan W, Tu S (2009) Influence of annealing on thermoelectric properties of bismuth telluride films grown via radio frequency magnetron sputtering. Thin Solid Films 517: 3731

Hudaka NS, Amatucci GG (1993) Small-scale energy harvesting through thermoelectric, vibration, and radiofrequency power conversion. J Appl Phys 103:101301

Hall K (2009) http://www.motorauthority.com/news/1024172_earlylook-at-bmws-next-wave-of-efficientdynamics-technology. Accessed 3 July 2012

Inayat SB, Hussain MM (2012) Thermoelectricity for green building exterior glasses, 31st International and 10th European Conference on Thermoelectrics. Aalborg, Denmark

Khatri H, Marsillac S (2008) The effect of deposition parameters on radiofrequency sputtered molybdenum thin films. J Phys Condens Matter 20:055206

Kishi M, Nemoto H, Hamao T, Yamamoto M, Sudou S, Mandai M and Yamamoto S (1999) Micro thermoelectric modules and their application to wristwatches as an energy source. In: Proceedings of the Eighteenth International Conference on Thermoelectrics, p 301

Rowe DM, Morgan DV, Kiely JH (1989) Miniature low-power/ highvoltage thermoelectric generator. Electron Lett 25:166

Snyder GJ, Lim JR, Huang CK, Fleurial JP (2003) Thermoelectric microdevice fabricated by a MEMS-like electrochemical process. Nat Mater 2:528

Song Y (2008) Oxide based thermoelectric materials for large scale power generation, M.S. Thesis, Dept. of Material Sciences and Engineering, Massachusetts Institute of Technology, Cambridge, MA

Yang J, Caillat T (2006) Thermoelectric materials for space and automotive power generation, Mater Res Bull 31:224 